\section{Haemodynamic monitoring: measurement of systemic blood pressure}

W. Andrew C. Scott MB BS FFARCS
No single clinical sign in the practice of anaesthesia is as important as the systemic blood pressure (BP). Knowledge of the true BP is essential in maintaining the milieu interior during surgery and it is used to "fine-tune" the depth of anaesthesia. Arterial catheterisation for direct measurement of BP by electromanometers provides clear advantages, the only major concern being the safety of arterial cannulation. Studies now confirm its safety but also have identified difficulties in measurement systems. Improved methods of indirect BP measurement are available but have not been subjected to full critical appraisal. This article reviews current knowledge concerning these techniques and recommends its application to clinical measurement of systemic blood pressure.

\section{Indirect methods}

Indirect methods require the use of a pressure cuff to occlude a major limb vessel and a method of detecting changes in arterial wall motion beneath the cuff or in flow distal to the cuff. The American Heart Association (AHA) publishes Recommendations for Human Blood Pressure Determination by Sphygmomanometers' which discusses common errors and recommends standard procedures. Optimum cuff size and the criterion for diastolic BP (BPDia) have been continuing topics for discussion. Observer factors such as digit preference, knowledge of previous readings and inattentiveness have been found to influence BP measurements. They are probably important factors. when frequent serial BP measurements are made. Too rapid cuff deflation ( $>0.5 \mathrm{kPa} / \mathrm{sec}$ ) can cause systematic underestimation of BP. Cuff size is critical in order to ensure the correct length of artery is occluded. The bladder width should be more than 40 per cent and less than 70 per cent of arm circumference while the length should cover at least 60 per cent. The "range marks" printed on the cuff provide a ready guide to correct sizing. In the lateral or sitting position indirect BP measurements are influenced both by hydrostatic pressure differences and other factors. In the lateral position up-arm cuff pressures are lower than aortic but down-arm cuff pressures may not be increased. ${ }^{2}$

Automation of indirect BP measurements provides several advantages. Readings are regularly updated during the "busy periods" at induction and emergence. Cuff deflation can be precisely controlled and linked to heart rate. Observer bias and fatigue are eliminated. Oscillometric methods can employ non-linear amplifiers and extrapolation to obtain better estimates of the null defiection points at systolic BP (BPSys) and BPDia than a human observer. Most semi-automatic and automatic sphygmomanometers use a piezoelectric microphone to transduce the Korotkov sounds (K-sounds) or oscillometry or a combination of both.

Inexpensive instruments designed for home use may be a cost-effective alternative to the traditional sphygmomanometer during anaesthesia. Cuff pressure and the amplitude of filtered $\mathrm{K}$-sounds are digitized and stored during cuff deflation. The data are scanned to determine the cuff pressure at which characteristic changes in frequency content occurred. Few published studies have examined their accuracy, none of these during anaesthesia. The

From the Departments of Anaesthesia, McGill University and Montreal General Hospital, 1650 Cedar Ave, Montreal, Quebec H3G 1 A4. 
TABLE I Mean Squared Difference of Paired Observations

\begin{tabular}{lrc}
\hline & BPSys & BPDia \\
\hline Dinamap & 6.79 & 5.53 \\
Yamasu & 5.08 & 4.63 \\
F ratio & 1.34 & 1.19 \\
Dinamap variability $>$ Yamasu & $\mathrm{p}<.05$ & $\mathrm{NS}$ \\
Health TOP & 114.45 & 65.97 \\
Dinamap & 69.30 & 37.11 \\
F ratio & 1.65 & 1.70 \\
Health TOP variability $>$ Dinamap & $\mathrm{p}<.0 \mathrm{t}$ & $\mathrm{p}<.01$ \\
\hline
\end{tabular}

variability (measured as the mean squared difference of duplicate measurements) using either the Yamasu (168 observations in 20 subjects) or Health-TOP (170 observations in 18 subjects) compared with simultaneous measurements made with a Dinamap 845 on the contralateral arm is shown in Table I. It suggests that the variability of the Yamasu (cost \$150) may be no greater than that of the Dinamap.

These sphygmomanometers are able to eliminate observer errors caused by improper deflation rate, digit preference, fatigue or background noise. They can be used without access to the arm though need to be checked occasionally by auscultation or palpation. Disadvantages include the interference caused by electrocautery, lightweight plastic cases (soluble in enflurane!) and a frustrating internal logic designed to protect naive users. Some fully automatic systems (eg. Cardivan 9200, Paramed and Vitatek 512) combine K-sound detectors and oscillometry. Using $\mathrm{K}$-sounds allows faster cuff deflation and is less prone to interference from arm movements. It is preferred by conscious patents who find the inflated cuff uncomfortable. The method requires accurate placement of the microphone which cannot be easily readjusted during surgery.

Oscillometry (the Flicker Method) has been used by anaesthetists for years to record BPSys. The main disadvantage is the lack of agreed criterion for BPSys. Although the maximum oscillations are generally taken to indicate mean arterial pressure, ${ }^{3}$ Bruner $^{4}$ found that they occur at BPSys. Even when performed under ideal conditions (new cuff, short tubing, anaeroid manometer with a low-friction bearing), there seem to be enough inter-observer differences to make the method useful for observing changes but not absolute values. The oscillometric method was further developed to measure mean arterial pressures. Mauck et $a l^{3}$ examined the relationship between the point of maximal oscillations (PMO) and mean arterial pressure in both mathematical and experimental models. They found a generally close relationship between PMO and BPMean over a wide range of input values. Both PMO and the changes in amplitude of the oscillations were decreased when the residual cuff volume was large. As pulse pressure decreases (eg. during hypotension) the PMO becomes a better estimator of BPMean. Both these findings led to its use initially for BP measurements in infants. This study did not examine the relationship between BPSys, BPDia and oscillatory cuff pressure, nor have the criteria for their determination been fully published. A number of studies have confirmed that there is a consistent relationship between measurements made with the Dinamap 845 system and direct arterial pressure measurements in both adults and infants when BPMean ranges between 6.7 and $16 \mathrm{kPa}$ (50- 120 torr). Although the overall relationship is close, the $95 \%$ confidence limits for single observations are at least $\pm 2 \mathrm{kPa}$ (15 torr). ${ }^{5}$

\section{Direct methods}

Direct arterial blood pressure measurement has never been easier. Monitors which require infrequent calibration and use digital zero subtraction can be set up in moments. Disposable monitoring kits have eliminated the problem of sterilizing transducers though apparently without completely eliminating the spread of nosocomial infection. ${ }^{6}$ The widespread use of controlled flush devices has reduced the hazard from retrograde embolization but does not seem to affect the incidence of post-cannulation thrombosis. ${ }^{7}$ Allen's test for incomplete collateral circulation in the hand is recommended prior to radial or ulna artery cannulation yet its use seems of doubtful significance. The results of two large series in which the Allen's test was ${ }^{8}$ or was not ${ }^{7}$ used as a guide to the site of cannulation demonstrate neither a marked difference in post-cannulation flow abnormality nor in ischemic complications. The risk of complications has been related to a number of factors such as size and shape of cannula, duration of cannulation, the use of vasopressors and prolonged low-flow states. The most recent, large scale and prospective study ${ }^{7}$ concludes that the risk of partial or complete 
occlusion of the artery after cannulation is more than $25 \%$. No important sequelae of such thrombosis occurred, nor was there evidence incriminating a particular type of cannula or method of insertion, nor was Allen's test predictive of ischemic changes after cannulation. Persistent abnormal sensation in the thumb occurred in one case out of 1699 . This would seem to confirm the general experience concerning the safety of arterial cannulation. Accidents caused by inadvertent injection of drugs intended for intravenous administration will continue to be an occasional hazard which could be eliminated by the introduction of non-interchangeable tapers for stopcocks in intravenous and intra-arterial lines.

Whilst continuous display of the arterial pressure waveform can be considered a low-risk procedure the technical problems concerning representation and interpretation of arterial pressure waveforms have not diminished. Bruner ${ }^{4}$ discussed the changes to the pressure pulse which occur as it travels from the aortic root to transducer diaphragm. These are caused by the differing transmission characteristics of the arterial system and the measuring system. The pressure pulse has two parts which may often be seen as twin peaks. The initial portion of the pressure pulse is caused by sudden acceleration of blood in the ascending aorta. Both the rate of increase and the peak value are related to aortic blood-flow acceleration and therefore indirectly reflect the contractile state of the left ventricle. The second part of the pressure wave is the volume displacement phase. This lasts longer and its size is related to the stroke output of the heart. As the pressure pulse travels from central to peripheral arteries part of the energy contained in the first part of the wave is reflected and summates with the next part of the pressure wave. The peak pressure is progressively delayed and increased in amplitude. These changes occur because the impedance of the peripheral arteries is greater than the central. An example of an abrupt change in impedance occurs when the arterial cannula is wide enough to occlude the artery. In this case the impedance suddenly becomes infinite, the total energy contained in the pressure wave is reflected and a large local increase in pulse amplitude occurs. A smaller cannula or a larger artery will reduce the amount of energy that is reflected.
The pressure peak also may be increased because of the characteristics of the external measuring system. Although some monitors incorporate electronic filters which can alter waveforms, the main external source of distortion is the hydrodynamic performance of the catheter-transducer system. This is characterised by two interactive factors, the natural frequency and damping coefficient. When natural frequency is high, clinically acceptable measurements are obtained despite wide variations in the damping coefficient. Conversely when natural frequency is low, results will be unacceptable regardless of the damping coefficient. ${ }^{9}$ The exact limits depend on the shape (and therefore frequency content) of the original waveform. Above $7 \mathrm{~Hz}$ the lower the natural frequency the nearer critically damped the system must be to allow clinically useful measurements. Unless the tubing is kept very short, accentuation of the early component of the pressure pulse can be expected. When natural frequency is very low, marked attenuation of the high frequency wave components occurs. This is the "damped" waveform seen when a large bubble is placed in the system and results in underestimation of BPSys. Table II shows the range of errors in BPSys which may be present when using some commercially available monitoring kits. These were obtained with a pressure waveform generator (Biotek Model 601), 20 ga cannula and Bell and Howell 4-327-I transducer and are similar to other previous observations. ${ }^{9,10}$ In $3 / 4$ systems tested, increasing heart rate without changing BPSys, increased peak pressure by at least 10 torr.

When a pressurized, continuous flush device is used to prime the tubing, air bubbles are formed by turbulent mixing of the air and flush solution in the drip chamber. Such bubbles "cling" tenaciously to the surface of the tubing. Further rapid flushing in an attempt to dislodge them aggravates the problem. To minimize bubbles the drip chamber should be as full as possible and the tubing primed using gravity before pressurizing the reservoir. " The mating of the disposable dome and the transducer diaphragm may cause an air bubble to be trapped between the dome and diaphragm. This greatly decreases the system's modulus of elasticity (i.e. the pressure change per unit change in volume) and decreases natural frequency. If an air bubble is present, the increased movements of the membra- 
using $\mathrm{K}$-sounds or oscillometry to determine BP need to be examined. Questions need to be asked about the algorithms used to determine BP parameters automatically so that their implications can be tested. It is to be hoped that the AHA or International Standards Organisation will adopt standards for automatic sphygmomanometers.

\section{References}

1 Kirkendall WN, Feinleib $M$, Freis ED, Mark $A L$. Recommendations for human bload pressure determination by sphygmomanometers Circulation 1980; 62: 1145A-55A.

2 Newton KM. Comparison of aoric and brachial cuff pressures in flat supine and lateral recumbent positions. Heart Lung 1981; 10: 821-6.

3 Mauck GW, Smith CR, Geddes LA, Bourland $I D$. The meaning of the point of maximum oscillations in cuff pressure in the indirect measurement of blood pressure - Part II. J Biomech Eng 1980; 102: 28-33.

4 Bruner JMR. Handbook of Blood Pressure Monitoring. Littleton, MA: PSG Publishing, Inc., 1978.

5 Hutton P, Dye J, Prys-Roberts C. An assessment of the Dinamap 845. Anaesthesia 1984; 39: 261-7.

6 Stratton $\mathrm{CW}$. Bacterial infection from intravascular monitoring devices. Infect Control 1983; 4: 399401.

7 Slogoff S, Keats AS, Ariund C. On the safety of artery cannulation. Anesthesiology 1983; 59: 42-7.

8 Mandel MA, Dauchot PJ. Radial artery cannulation in 1000 patients: Precautions and complications.

J Hand Surg 1977; 2: 482-5.

9 Gardner RM. Direct blood pressure measurement Dynamic response requirements. Anesthesiology 1981; 54: 227-36.

10 Miller GS, Zhiblut JP. Practical evaluation of catheter - transducer coupling systems for artifact. Heart Lung 1983; 12: 156-61.

11 Gardner RM, Bond EL, Clark JS. Safety and efficacy of continuous flush systems for arterial and pulmonary artery catheters. Ann Thor Surg 1977; 23: 534-48. 